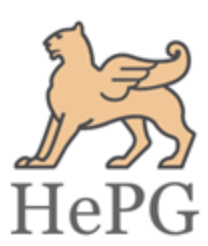

ISSN: 2348-1900

Plant Science Today

http://www.plantsciencetoday.online

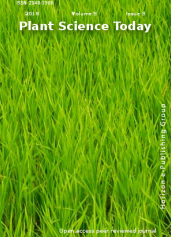

OPEN ACCESS

Research Communication

\title{
Assessment of diurnal variation in Ocimum sanctum Linn. by gas chromatographic fingerprint analysis coupled with chemometric methods
}

\author{
"Nikunj Patel, Niranjan Kanaki, Vinit Movaliya \\ K.B. Institute of Pharmaceutical Education and Research, Kadi Sarva Vishwavidyalaya, Gandhinagar - 382023, India
}

\section{Article history}

Received: 27 July 2018

Accepted: 20 August 2018

Published: 02 September 2018

\section{Editor}

Raul Rodrguez-Herrera, Universidad Autonoma de Coahuila, Saltillo, Mexico

\section{Publisher}

Horizon e-Publishing Group

\author{
*Correspondence \\ Nikunj Patel \\ 凶ndthummar@gmail.com
}

\begin{abstract}
Vast intra-specific variations, especially diurnal, geographical and seasonal, have been reported in the chemical composition of essential oils of Ocimum species. The study was conducted to assess diurnal variation in the chemical composition of the leaves of Ocimum sanctum. The leaf samples collected at different times of the day were analyzed by gas chromatography coupled with flame ionization detector (GC-FID). The chromatographic fingerprints of different leaf samples were analyzed by chemometric methods like principal component analysis and hierarchical cluster analysis. No significant difference was found in the chemical compositions of the leaf samples collected at different times of the day. The results lead to a conclusion that $O$. sanctum does not exhibit diurnal variation in its chemical composition, unlike O. gratissimum.
\end{abstract}

\section{Keywords}

Ocimum sanctum; diurnal variation; chemometric method; essential oil composition; GC-FID

\section{Citation}

Patel N, Kanaki N, Movaliya V. Assessment of diurnal variation in Ocimum sanctum Linn. by gas chromatographic fingerprint analysis coupled with chemometric methods. Plant Science Today 2018;5(3):131-134. https://dx.doi.org/10.14719/pst.2018.5.3.413

Copyright: ( $)$ Patel et al (2018). This is an open-access article distributed under the terms
of the Creative Commons Attribution License, which permits unrestricted use, distribution,
and reproduction in any medium, provided the original author and source are credited and reproduction in any medium, provided the
(https://creativecommons.org/licenses/by/4.0/).

\section{Introduction}

The genus Ocimum has enormous significance due to the medicinal and aromatic properties and its uses as spices. However, vast intra-specific variations have been reported in various species, with respect to morphological features and chemical composition. In a study conducted to assess the extent of diurnal variation in the essential oil composition of Ocimum gratissimum, drastic changes in the contents of eugenol and 1,8- cineole were observed throughout the day time [1]. Similarly, significant diurnal variations were also observed in the yield and composition of essential oil in Ocimum basilicum, Ocimum kilimandscharicum and Ocimum americanum [2]. Significant differences were found in total content and composition of essential oils between young and mature leaves of $O$. basilicum [3]. Significant seasonal and geographical variations were observed in the composition of essential oils of Ocimum sanctum and O. basilicum, and these 


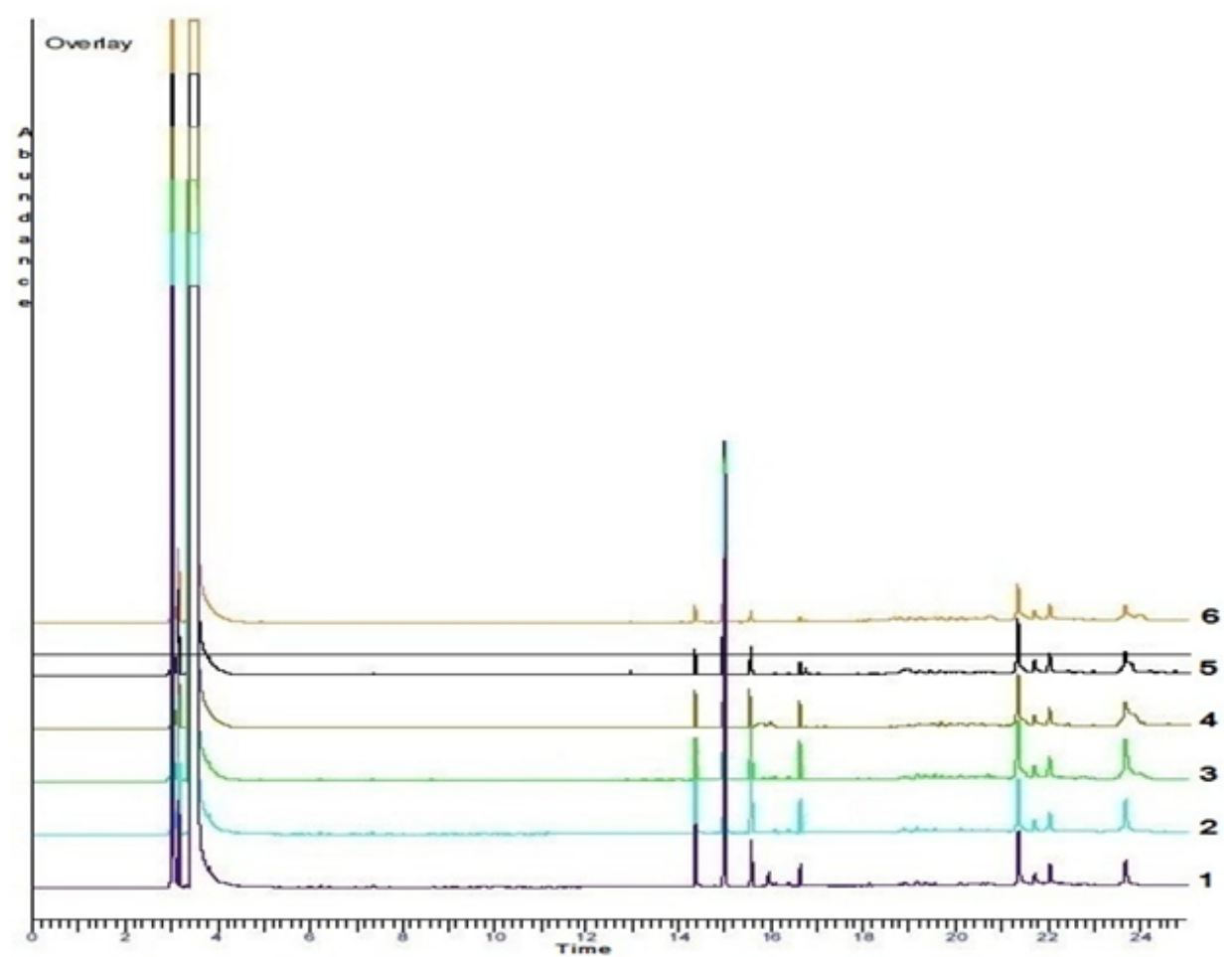

Fig. 1. Gas chromatographic fingerprint profile of Ocimum sanctum leaf samples (1-6) collected at six different time points of the day during 8 a.m. to 6 p.m.

variations could form the basis for deciding the appropriate season and geographical location for collection of these species [4, 5]. However, diurnal variation in the composition of essential oil of $O$. sanctum has not been studied so far. Considering the importance of $O$. sanctum in the Indian tradition, in which it is considered to be sacred and having huge therapeutic potential with activities like anti bacterial, anti carcinogenic, anti oxidant, neuro-protective, anti stress, cardio protective etc. [6], such investigation would help in predicting the appropriate time of collection of leaves to make the most of its medicinal and aromatic potential. This paper reports the analysis of Ocimum sanctum leaves for investigation of diurnal variation in its chemical composition. In this paper the results of analyses of the $O$. sanctum leaves collected at elapsing daytime, during an entire day, are reported.

\section{Materials and Methods}

The leaves of $O$. sanctum were collected for the study from plants growing in Gandhinagar (N 2312'56.29”; E 72³8'12.99”) Gujarat, India, situated at $\sim 78.93 \mathrm{~m}$ above sea level. The plant was identified by a taxonomist and a herbarium specimen was deposited in the Pharmacognosy department of our institute. The leaf samples were collected in the month of May for three successive days from Gandhinagar, Gujarat, where the sun rises at $05.45 \mathrm{a}$.m. and sets at $07.00 \mathrm{p} . \mathrm{m}$. during the month. The samples were collected between 8 a.m. and 6 p.m. at regular intervals of $2 \mathrm{hrs}$ each. For extraction of plant material, one gram powder of dried leaves of $O$. sanctum was soaked overnight with $25 \mathrm{~mL}$ chloroform in a $100 \mathrm{~mL}$ conical flask. The extract was filtered through Whatman No. 1 filter paper, refluxed and concentrated on a water bath at $60^{\circ} \mathrm{C}$. The final volume of the concentrated extract was adjusted to $5 \mathrm{~mL}$. This extract was used for gas chromatographic analysis. The analysis of extract was achieved using Perkin Elmer GC Clarus 500 equipped with a fused silica ZB-5capillary column (30 m $\times 0.25 \mathrm{~mm}$; $0.25 \mu \mathrm{m}$ film thickness) and flame ionization detector. The carrier gas was nitrogen at $1.0 \mathrm{~mL} / \mathrm{min}$ flow rate. The initial oven temperature was $60^{\circ} \mathrm{C}$ which was raised to $220^{\circ} \mathrm{C}$ with $8.0^{\circ} \mathrm{C} / \mathrm{min}$ ramp rate and was held at that temperature for $5 \mathrm{~min}$. The injector and detector temperatures were $250^{\circ} \mathrm{C}$ and $260^{\circ} \mathrm{C}$, respectively. The injection volume of the sample was $2.0 \mu \mathrm{L}$. The sample was injected using a split ratio of 20:1. Average chromatographic fingerprint data of three successive days respective to their time point collection was subjected to statistical analysis using Minitab 17 software. Each sample was processed and analyzed in triplicate and the results were analyzed by two-way ANOVA to assess the diurnal variation in the chemical composition of leaves. A value of $p<0.05$ was considered to indicate statistically significant difference among the samples. For further discriminating the investigated samples, principal component analysis (PCA) [7, 8], was carried out based on the differences in the samples. PCA was carried out on the data of areas of common peaks obtained from the GC fingerprints of different samples. In order to evaluate the resemblance and differences in the leaf samples collected at different time points, hierarchical cluster analysis (HCA) was performed on the data of areas of common peaks in the GC 
Table 1. Gas chromatographic data of Ocimum sanctum leaf samples collected at different times of the day

\begin{tabular}{cccccccccccc}
\hline $\begin{array}{c}\text { Time of } \\
\text { collection }\end{array}$ & \multicolumn{10}{c}{ Peak Area(\%) at retention time (min.) } \\
\cline { 2 - 12 } & $\mathbf{1 4 . 3}$ & $\mathbf{1 5 . 0}$ & $\mathbf{1 5 . 6}$ & $\mathbf{1 6 . 0}$ & $\mathbf{1 6 . 6}$ & $\mathbf{1 8 . 9}$ & $\mathbf{1 9 . 5}$ & $\mathbf{2 1 . 3}$ & $\mathbf{2 1 . 7}$ & $\mathbf{2 2 . 0}$ & $\mathbf{2 3 . 7}$ \\
\hline 8 a.m. & 5.90 & 57.15 & 5.74 & 0.82 & 4.71 & 1.87 & 0.71 & 8.89 & 1.96 & 3.01 & 2.52 \\
10 a.m. & 5.71 & 56.96 & 5.81 & 0.87 & 4.82 & 1.79 & 0.59 & 8.64 & 1.84 & 2.89 & 2.4 \\
12 p.m. & 5.89 & 57.01 & 5.71 & 0.82 & 4.83 & 1.97 & 0.65 & 8.92 & 1.74 & 2.91 & 2.51 \\
2 p.m. & 5.69 & 56.94 & 5.62 & 0.84 & 4.69 & 1.91 & 0.62 & 8.88 & 1.98 & 2.85 & 2.89 \\
4 p.m. & 5.92 & 56.61 & 5.78 & 0.89 & 4.75 & 2.02 & 0.64 & 8.73 & 2.02 & 2.02 & 2.71 \\
6 p.m. & 5.91 & 57.00 & 5.89 & 0.88 & 4.81 & 1.98 & 0.61 & 8.69 & 1.97 & 2.09 & 2.67 \\
\hline
\end{tabular}
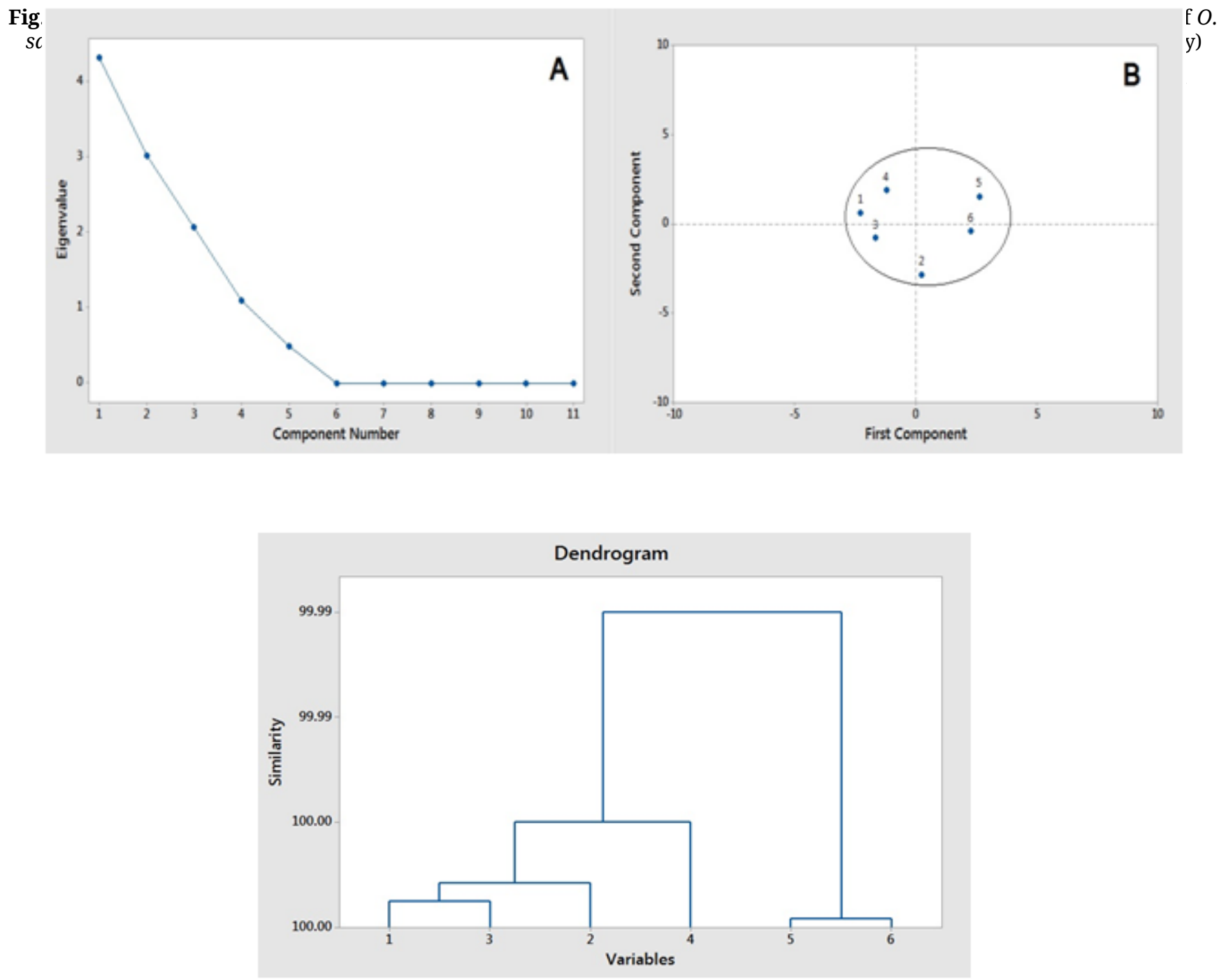

fingerprints. HCA is a multivariate analysis technique that can be used to divide samples into groups.

\section{Result and Discussion}

The leaves of $O$. sanctum were collected for three successive days in the month of May, since the solar intensity and temperature are highest and their diurnal fluctuations are more prominent during this month as compared to other months of the year. Hence, it was thought to be the ideal time of the year to study diurnal variation in $O$. sanctum.
Chloroform was used as a solvent for extraction of leaf samples, since most of the volatile oil components are soluble in it. A more polar solvent like methanol would also dissolve volatile oil components, but many other non-volatile polar compounds will also get dissolved in it and will interfere with the GC analysis. All the extraction and concentration procedures were performed at temperature not exceeding $60^{\circ} \mathrm{C}$ to avoid loss of volatile constituents. A chromatographic method was developed for GC fingerprint analysis of extracts of leaf samples. The relative percentage contents of components of the extracts were 
determined from the relative areas of the peaks resolved in the gas chromatogram. The GC fingerprint profile of the chloroform extract of $O$. sanctum leaves showed 11 prominent peaks, which were considered for further statistical analysis of the fingerprint data. The average retention times and peak areas of chromatographic fingerprint analysis of leaf samples collected on three successive days was used for statistical analysis. The average of chromatographic data and overlain chromatograms of all the six samples of a day are shown in Table 1 and Fig. 1, respectively. Statistical analysis of GC fingerprint data, performed using two-way ANOVA, revealed that there was no significant difference in the composition of the leaf samples $(p>0.05)$ collected at different time points of the day. Thus, no apparent diurnal variation was found in the chemical composition of leaves of $O$. sanctum. The fingerprint data obtained from GC analysis of leaf samples can be easily distinguished and discriminated using a dimensionality reduction method such as PCA [9]. PCA of the GC fingerprint data of different leaf samples was carried out using correlation type of matrix and all data were displayed as scores and loadings in a coordinate system of principal components resulting from data dimensionality reduction. It was found that the first four principal components (PC 1-4) accounted for over $95.7 \%$ of the variation, as shown in the scree plot (Fig. 2-A). The score plot for different leaf samples revealed no significant differences amongst the samples, as evident from the close distribution of the samples in the plot (Fig. 2-B). Thus, PCA of fingerprint data of $O$. sanctum leaf samples does not suggest any diurnal variation in the chemical composition of the leaves. A hierarchical agglomerative clustering analysis (HCA) of six samples of $O$. sanctum leaves collected at different time points was performed after normalizing the data, to visualize the differences and/or similarities among samples. In HCA analysis (Fig. 3), the six leaf samples got distributed into two clusters; cluster-I contained samples 1, 2, 3 and 4 and cluster-II contained samples 5 and 6 . However, the similarity level of these two clusters was almost $99.99 \%$, indicating that all the six samples were almost similar to each other. The results obtained after hierarchical cluster analysis are in agreement with those obtained after principal component analysis and ANOVA.

\section{Conclusion}

Though diurnal variation has been reported in the chemical composition of the essential oil from $O$. gratissimum [1], which is taxonomically very close to $O$. sanctum, no significant diurnal variation was found in the chemical composition of the later species. The results of the study indicate that the variation in the time of collection of the leaves of $O$. sanctum may not have significant influence on the composition of its essential oil and hence, on its medicinal properties attributable to its essential oil composition.

\section{Competing Interests}

There is no conflict of interest among the authors.

\section{Acknowledgments}

This work is a part of research project assisted by the INSPIRE Programme division (Department of Science and Technology - DST, Government of India, New Delhi). The authors are thankful to the DST for the same.

\section{Author's contributions}

All the authors contributed equally to prepare the article.

\section{References}

1. de Vasconcelos Silva MG, Craveiro AA, Matos FA, Machado MI, Alencar JW. Chemical variation during daytime of constituents of the essential oil of Ocimum gratissimum leaves. Fitoterapia. 1999; 70(1):32-4. https://doi.org/10.1016/ $\underline{\text { S0367-326X(98)00020-3 }}$

2. Padalia RC, Verma RS, Chauhan A. Diurnal variations in aroma profile of Ocimum basilicum L., O. gratissimum L., $O$. americanum L., and $O$. kilimandscharicum Guerke. Journal of Essential Oil Research. 2017; 29(3):248-61. https://doi.org/10.1080/10412905.2016.1216898

3. Chang X, Alderson PG, Wright CJ. Variation in the essential oils in different leaves of basil (Ocimum basilicum L.) at day time. The Open Horticulture Journal. 2009; 2:13-6. http://dx.doi.org/10.2174/1874840600902010013

4. Vani SR, Cheng SF, Chuah CH. Comparative study of volatile compounds from genus Ocimum. American Journal of Applied Sciences. 2009; 6(3):523. https://doi.org/10.2174/1874840600902010013

5. Zheljazkov VD, Cantrell CL, Evans WB, Ebelhar MW, Coker C. Yield and composition of Ocimum basilicum L. and Ocimum sanctum L. grown at four locations. HortScience. 2008; 43(3):737-41. https://doi.org/10.1021/jf072447y

6. Rahman S, Islam R, Kamruzzaman M, Alam K, Jamal AH. Ocimum sanctum L.: A review of phytochemical and pharmacological profile. American Journal of Drug Discovery and Development. 2011:1-5. https://doi.org/10.4103/0973-7847.65323

7. Kannel PR, Lee S, Kanel SR, Khan SP. Chemometric application in classification and assessment of monitoring locations of an urban river system. Analytica Chimica Acta. 2007; 582(2):390-9. https://doi.org/10.1016/j.aca.2006.09.006

8. Pierce KM, Hope JL, Johnson KJ, Wright BW, Synovec RE. Classification of gasoline data obtained by gas chromatography using a piecewise alignment algorithm combined with feature selection and principal component analysis. Journal of Chromatography A. 2005; 1096(12):101-10. https://doi.org/10.1016/j.chroma.2006.06.087

9. Chen Y, Zhu SB, Xie MY, Nie SP, Liu W, Li C, Gong XF, Wang YX. Quality control and original discrimination of Ganoderma lucidum based on high-performance liquid chromatographic fingerprints and combined chemometrics methods. Analytica Chimica Acta. 2008 Aug 15; 623(2):146-56. https://doi.org/10.1016/j.aca.2008.06.018 